\title{
КВАЗИСОСРЕДОТОЧЕННЫЕ РЕАКТИВНЫЕ ЭЛЕМЕНТЫ НА ОСНОВЕ КРИСТАЛЛОПОДОБНЫХ НЕОДНОРОДНОСТЕЙ
}

\author{
БИДЕНКО П.С., НЕЛИН Е.А., НАЗАРЬКО А.И., АДАМЕНКО Ю.Ф. \\ Национальный технический университет Украинь \\ «Киевский политехнический институт», \\ Украина, Киев, 03056, пр-т Победы 37
}

\begin{abstract}
Аннотация. Предложена реализация квазисосредоточенных реактивных элементов электромагнитнокристаллическими (ЭК) неоднородностями. Выполнено сравнение характеристик ЭК-неоднородностей и традиционных микрополосковых структур, иллюстрирующее существенное увеличение (в 1,5 ...4 раза) значений реактивностей ЭК-неоднородностями. Моделирование выполнено в программном пакете CST Microwave Studio. Приведены экспериментальные характеристики для предложенной и традиционной квазисосредоточенных индуктивностей. Проанализирована погрешность параметра квазисосредоточенного элемента и показана возможность ее минимизации
\end{abstract}

Ключевые слова: микрополосковая структура; электромагнитнокристаллическая неоднородность; квазисосредоточенный реактивный элемент

\section{ВВЕДЕНИЕ}

Микрополосковые устройства широко применяются в радиоэлектронных системах различного назначения. В последнее время в микрополосковой технике сложились направления на основе новых технологических решений и материалов, позволяющих существенно уменьшить габариты устройств и повысить их избирательность [1].

Одно из этих направлений - искусственные структуры с особыми характеристиками $[1,2]$. К таким структурам относят кристаллоподобные структуры с зонными свойствами, аналогичными кристаллам [2]. В микрополосковых устройствах применяют микрополосковые кристаллоподобные структуры - электромагнитные кристаллы (ЭК), а также отдельные ЭК-неоднородности. Традиционные ЭК-неоднородности выполняют двухмерными в виде отверстия или щели разной формы в ме- таллизированной поверхности или в сигнальном проводнике [1, 3, 4].

В микрополосковых структурах используются элементы с низким $\left(Z_{\mathrm{H}}\right)$ и высоким $\left(Z_{\mathrm{B}}\right)$ волновыми импедансами $[1,5]$. Предельные значения величин $Z_{\mathrm{H}}, Z_{\text {в }}$ ограничены допустимой шириной сигнального проводника и составляют приблизительно 20 Ом и 120 Ом [6]. Традиционные двухмерные ЭК-неоднородности по своему характеру высокоимпедансные с эквивалентным волновым импедансом, не превышающим 210 Ом [7].

В [8-10] предложены высоко- и низкоимпедансные трехмерные ЭК-неоднородности, сочетающие неоднородности в металлизированной поверхности, в диэлектрике и на сигнальной поверхности, с заметно большими и меньшими эквивалентными импедансами. Например в [8] рассмотрена ЭК-неоднородность с $Z_{\text {в }}=360$ Ом, а в [9] с $Z_{\text {н }}=4$ Ом. 